\title{
When the Innocent Bystander Becomes the Culprit: Two Episodes of ST- Elevation Myocardial Infarction with Different Culprit Coronary Arteries in A Single Patient Occurring Two Days a Part
}

\section{Divinagracia Alban ADM*and Alimurung BN}

Makati Medical Center, Philippines

*Corresponding author: Divinagracia Alban ADM, Makati Medical Center, Philippines, Tel: +6328888999;

E-mail: aileen.divinagracia@yahoo.com

Rec Date: Jan 4, 2017, Acc Date: Jan 25, 2017, Pub Date: Jan 28, 2017

Citation: Divinagracia Alban ADM, et al. When the Innocent Bystander Becomes the Culprit: Two Episodes of ST-Elevation Myocardial Infarction with Different Culprit Coronary Arteries in A Single Patient Occurring Two Days a Part. Interv Cardiol J 2017, 3:1.

\section{Abstract}

Background: Recurrent ST elevation myocardial infarction (STEMI) with different culprit lesions in a single patient occurring few days apart is uncommon with limited similar cases reported.

Case Presentation: This is a case of a 53-year-old male who presented to the Emergency Department with left upper quadrant abdominal pain and an initial electrocardiogram showing ST elevation $\mathrm{MI}$ in the anterolateral leads. He underwent successful primary angioplasty of the culprit lesion (LAD) with placement of a single Everolimus drug-eluting stent with plans for staged intervention within a month of the remaining severe lesion in the left circumflex (LCX) artery (non-infarct related vessel). Two days after the initial STEMI, the patient experienced recurrence of abdominal pain with hypotension, and a repeat ECG revealed new ST segment elevation in the inferior leads. A repeat coronary angiogram demonstrated a widely patent stent in the LAD and the previously noted diseased LCX. No other new lesions were noted and the RCA was also patent. The patient then underwent a successful second primary $\mathrm{PCl}$ of the previously untreated severe lesion in the $L C x$.

Conclusion: Consecutive episodes of acute myocardial infarction in different coronary arteries occurring within hours to a few days after primary $\mathrm{PCl}$ for STEMI is uncommon and can be catastrophic if not detected early. The mechanism and predisposing factors to such event are still unclear. The optimum interventional treatment strategy regarding the immediate management of STEMI in patients with multi-vessel disease remains controversial.

Keywords: ST elevation myocardial infarction, Reinfarction, Infarct-related artery, Non-infarct related artery, Culprit lesion

\section{Case Report}

This is a case of a 53-year-old male, non-hypertensive, nondiabetic, heavy cigarette smoker, who was admitted to our institution for progressive, severe, left upper quadrant (LUQ) abdominal pain radiating to the upper back associated with nausea occurring one day prior to confinement. There was no chest pain, no dyspnea, and no diaphoresis. The patient went to the emergency department of another hospital, where an electrocardiogram (ECG) showed ST elevation in the anterolateral leads. Loading dose of dual anti-platelets (Aspirin $320 \mathrm{mg}$ and Ticagrelor $180 \mathrm{mg}$ ) were administered and the patient was advised to undergo emergency coronary angiogram with primary percutaneous intervention $(\mathrm{PCl})$. He was then transferred to our institution.

Upon arrival at our institution, the patient was not in distress and hemodynamically stable with persistent left upper abdominal pain. Pertinent physical exam findings include: blood pressure (BP) of 90/60, heart rate of $115 \mathrm{bpm}$, regular heart rhythm, grade $3 / 6$ holosystolic murmur at the left parasternal area, and S3 gallop sound. ECG showed ST segment elevation in leads V1-V5, I and avL (Figure 1).

Emergency left heart catheterization with coronary angiography revealed 2-vessel coronary artery disease with total $(100 \%)$ occlusion of the proximal left anterior descending (LAD) artery (Figure $\mathbf{2 A}$ ) and $80 \%$ to $90 \%$ discrete stenosis of the mid left circumflex (LCX) artery and $80 \%$ stenosis of the ostium of the first obtuse marginal (OM1) branch (Figure $2 \mathrm{C}$ ). Percutaneous transluminal coronary angioplasty (PTCA) of the LAD (culprit) lesion was performed with placement of a single $2.75 \mathrm{~mm} \times 24 \mathrm{~mm}$ drug-eluting (Everolimus) stent with resulting TIMI III flow to the distal LAD segment post-stenting (Figure 2B).

The plan was to continue dual antiplatelet medications and standard management for ST segment elevation myocardial infarction (STEMI) and to perform staged percutaneous coronary intervention $(\mathrm{PCl})$ of the remaining severe lesion of the $\mathrm{LCX}$ within one month.

2D echocardiogram done after the angioplasty showed concentric left ventricular (LV) remodeling with severe 
hypokinesia of the anterior and inferior interventricular septum and anterolateral LV free wall from mid to apex. The left ventricular ejection fraction (EF) was depressed at $46 \%$ by Simpson's method. The patient developed progressive shortness of breath and was managed with intravenous diuretics for acute decompensated heart failure secondary to anterior STEMI.

On the 3rd hospital day, the patient had recurrence of vague abdominal discomfort and hypotension (BP range of 80-90/50-60 with associated new ST elevation in lead II noted on the bedside cardiac monitor. A repeat 12-lead ECG showed new ST segment elevation in the inferior leads (Figure 3 ).

Dobutamine therapy was initiated. The patient underwent repeat emergency coronary angiogram which revealed that the previously stented proximal LAD was widely patent, with no signs of stent thrombosis and with TIMI III flow throughout the distal vessel (Figure 4A). The RCA was patent as well (Figure 4B) and the previously noted severe non-infarct lesions in the LCX and OM1 were unchanged (Figure 4C). No new lesion was found in the rest of coronary arteries. A $3.0 \mathrm{~mm} \times$ $20 \mathrm{~mm}$ drug-eluting (Everolimus) stent was deployed in the mid-LCX with TIMI III flow evident throughout the LCX poststenting (Figure 4D).

Maximal medical therapy for heart failure was continued. Patient experienced gradual improvement of the heart failure symptoms over the next few days and with downward trend of the cardiac enzymes. He was subsequently discharged symptom-free on the 12th hospital day. Nine months after the two $\mathrm{PCl}$ procedures, the patient is symptom-free and returned to full time work as a private company driver.

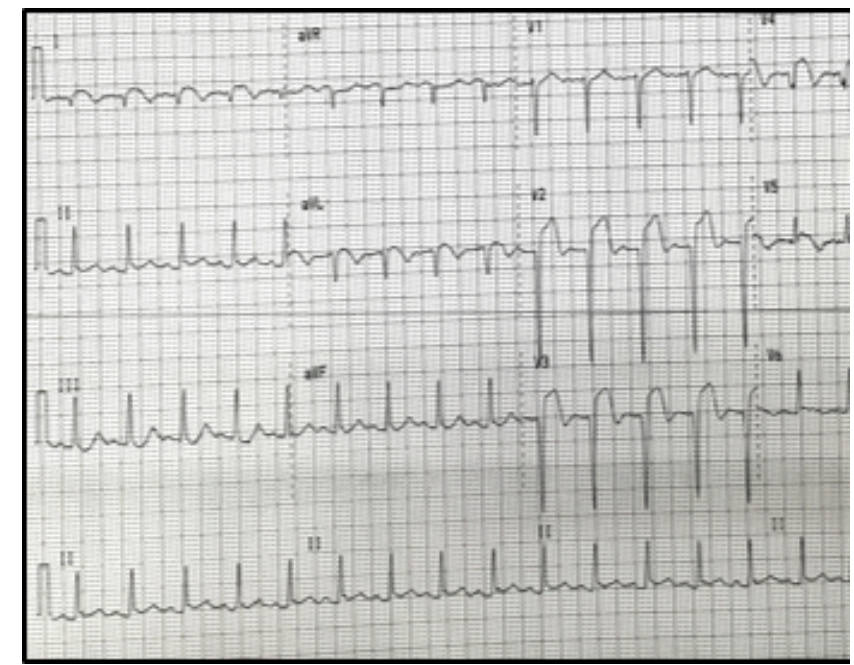

Figure 1 Admission ECG showing ST segment elevation on the anterolateral leads.

\section{Discussion}

There is ongoing debate regarding the optimum immediate management of ST segment elevation myocardial infarction (STEMI) in the setting of multi-vessel disease and whether primary $\mathrm{PCl}$ of "culprit-only" lesions versus "preventive" $\mathrm{PCl}$ of all significant lesions should be performed during the index $\mathrm{PCl}$.

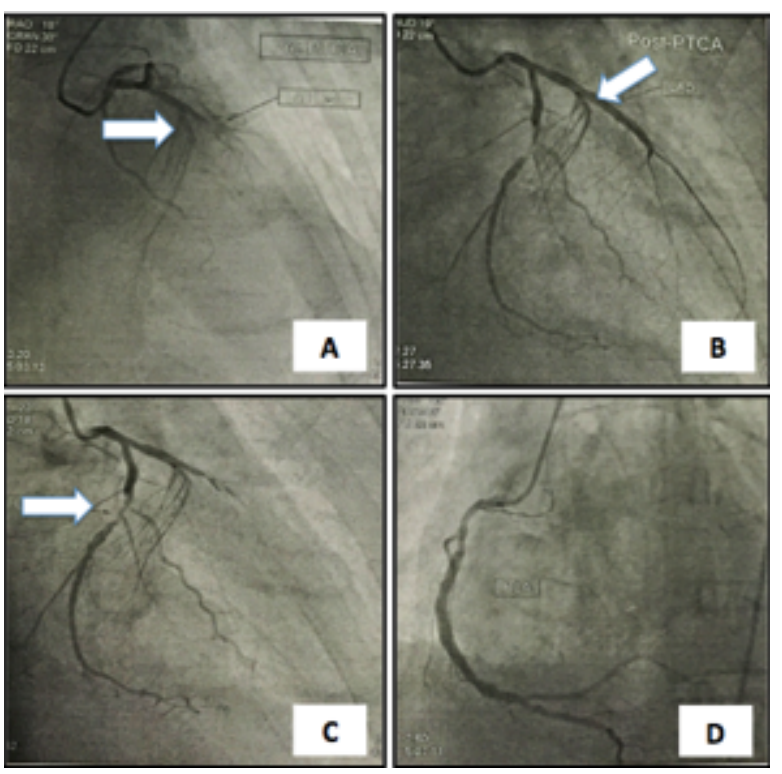

Figure 2 Coronary angiogram and angioplasty images on admission. A) Coronary angiogram showing totally occluded LAD. B) Restoration of TIMI III flow to the distal segment of LAD post-stenting. C) An $80 \%$ to $90 \%$ stenosis of the mid left circumflex (LCX) artery and $80 \%$ stenosis of the ostium of the first obtuse marginal (OM1) branch. D)Patent RCA with luminal irregularities.

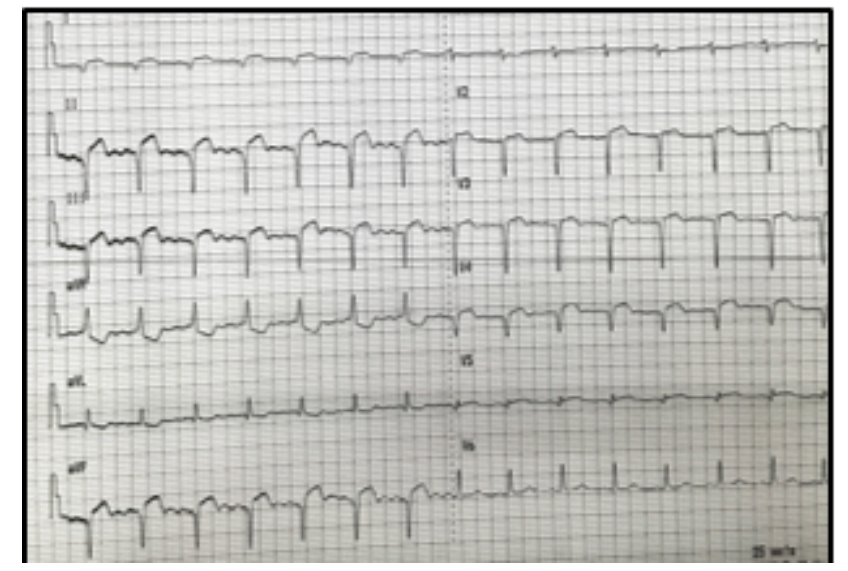

Figure 3 ECG on the second hospital day showing ST segment elevation in the inferior leads.

Prior guidelines $[1,2]$ recommend that $\mathrm{PCl}$ of the non-infarct related artery should not be performed at the time of index primary $\mathrm{PCl}$ in patients with STEMI who are hemodynamically stable, and that primary $\mathrm{PCl}$ should be limited to the culprit vessel unless there is continuing cardiogenic shock and persistent ischemia after successful $\mathrm{PCl}$ of the index culprit lesion. 


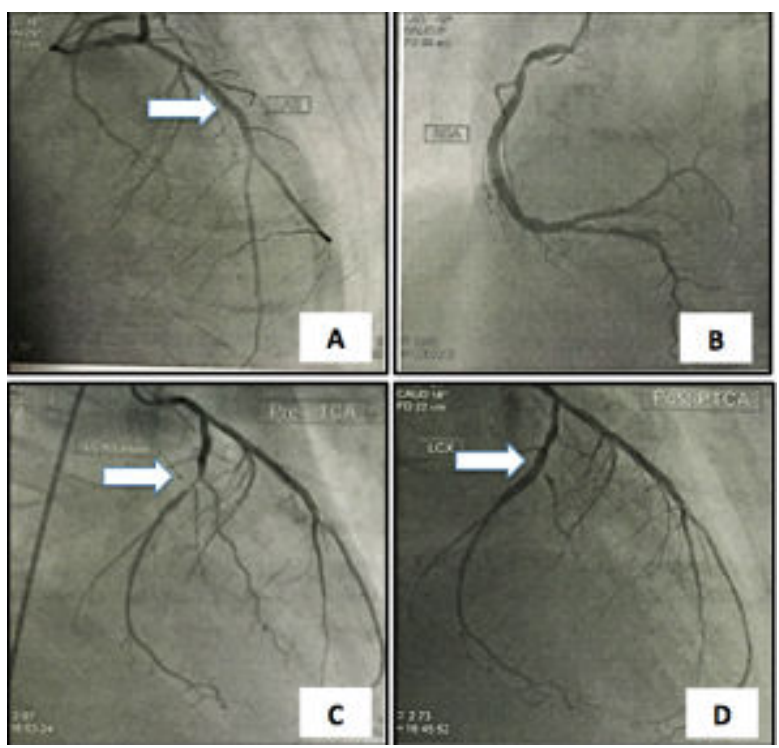

Figure 4 Coronary angiogram and angioplasty images 2 days post-PCl of the LAD. A) Coronary angiogram showing patency of the stent in the LAD with TIMI III flow to the distal segment. B) No significant lesion noted in the RCA. C) The untreated $80 \%$ to $90 \%$ stenosis in the mid left circumflex (LCX) artery was unchanged. D) Post-stenting of the LCX with TIMI III flow to the distal segments of the LCX.

However, several recent trials showed that $\mathrm{PCl}$ of the noninfarct related artery at the index admission resulted in a reduction in subsequent cardiovascular outcomes in STEMI patients. In 2013, Wald et al. [3] published the randomized trial of preventive angioplasty in myocardial infarction (PRAMI trial) where they enrolled 465 patients with multi-vessel disease with acute STEMI and randomly assigned patients to either infarct-artery $\mathrm{PCl}$ alone or preventive $\mathrm{PCl}$ of all severe lesions. They concluded that in patients undergoing emergency infarct-artery $\mathrm{PCl}$ for acute STEMI, preventive $\mathrm{PCl}$ of significant lesions including significant stenosis of noninfarct related arteries reduced the risk of subsequent adverse cardiovascular events, as compared with $\mathrm{PCl}$ limited to the infarct artery [3]. In 2015, the Complete Versus Lesion-Only Revascularization in Patients Undergoing Primary Percutaneous Intervention for STEMI (CVLPRIT Trial) was published, comparing complete revascularization versus treatment of infarct-related artery (IRA) only during the index admission. They enrolled 296 patients who underwent coronary angiography and were randomized to either inhospital complete revascularization or IRA-only revascularization. They concluded that in patients with multivessel disease presenting for primary $\mathrm{PCl}$, complete revascularization during the index admission significantly lowered the incidence of the composite primary endpoint (death, recurrent myocardial infarction, heart failure, ischemia-driven revascularization) at 12 months when compared with $\mathrm{PCl}$ of the IRA lesion alone [4]. In that same year, the DANAMI 3 PRIMulti Trial was also published, wherein the utility of infarct-related $\mathrm{PCl}$ vs FFR-guided complete revascularization were compared in multivessel STEMI patients. The authors of the said study concluded that in patients with multivessel disease presenting with STEMI and undergoing successful primary $\mathrm{PCl}, \mathrm{FFR}$-guided multivessel $\mathrm{PCl}$ with a goal of revascularization prior to hospital discharge is superior to culprit-only $\mathrm{PCI} 5$.

The above-mentioned trials led to the 2015 ACC/AHA/SCAI Focused Update revision on the previous 2013 ACC/AHA Guideline for the Management of STEMI and the 2011 ACC/AHA/SCAI guideline for percutaneous coronary intervention. Instead of a Class III (Harm) recommendation regarding multi-vessel $\mathrm{PCl}$ in hemodynamically stable patients with STEMI, the revised 015 guidelines upgraded the recommendation to a Class IIb Level of Evidence B for multivessel $\mathrm{PCl}$ to be performed either at the time of primary $\mathrm{PCl}$ or as a staged procedure during the index admission or at a later date [5]

The disadvantages of multi-vessel $\mathrm{PCl}$ during the index procedure include the increased risk for contrast-induced nephropathy, increased dose of radiation, increased risk of peri-procedural complications and increased risk of stent thrombosis. On the other hand, the disadvantages of doing culprit-only revascularization/staged $\mathrm{PCl}$ are the risk for recurrent angina, the risk of unstable lesions and a higher patient expense for another procedure [6]. The 2014 ESC guidelines on myocardial revascularization, recommend that multi-vessel PCl during STEMI during the index intervention should be considered in patients with cardiogenic shock in the presence of multiple, critical stenoses or highly unstable lesions (angiographic signs of possible thrombus or lesion disruption), and when there is persistent ischemia after $\mathrm{PCl}$ of the supposed culprit lesion [7].

In patients presenting with STEMI, multi-vessel coronary artery disease is found in $41 \%$ to $67 \%$ of patients depending upon the baseline characteristics (especially age) of the specific population studied [8]. However, to date, the challenge remains as to how to best identify patients who are most likely to benefit from preventive $\mathrm{PCl}$ and to what degree of lesion severity should warrant the management strategy?

In the case presented in this report, the attending interventional cardiologist opted to perform PCl of the culpritlesion alone as recommended in the current STEMI guidelines with a plan to perform staged $\mathrm{PCl}$ of the remaining significant stenosis within a month. In post-PCl patients who present with recurrence of symptoms and ST segment elevation, the most common identified cause is early in-stent restenosis/ thrombosis. The ST segment changes would typically be manifest in the same leads. However, in this case, the ST segment elevation 2 days post-PCI were in different leads (inferior) as compared with the initial ECG changes on admission (anterolateral), suggesting a new infarction in another myocardial territory, wherein the previous non-infarct related artery now became the culprit lesion, necessitating another emergency coronary angiogram with $\mathrm{PCl}$ of a second infarct-related artery. This occurrence is very rare with very limited similar case reports. 
In 2014, Chang et al. from Taiwan reported a case of an elderly hypertensive male presenting with acute chest pain and nausea with hemodynamic instability and an ECG showing ST elevation in the inferior leads. Loading dose of dual antiplatelets and vasopressor were initiated. The patient was found to have a subtotal occlusion of the RCA, with $50 \%$ stenosis of the LAD and LCX on coronary angiogram, and subsequently underwent $\mathrm{PCl}$ of the RCA. The patient's BP improved with vasopressors but had pulseless ventricular tachycardia and underwent defibrillation, intubation and IABP insertion. Repeat ECG showed ST elevation in V2-V6. Repeat coronary angiogram was performed revealing a patent RCA stent and new total occlusion of the mid-LAD. Thrombus aspiration and stenting of the mid-LAD was done with resulting TIMI III flow to the distal vessel. However, the unstable hemodynamic status persisted and the patient was found to have urosepsis and disseminated intravascular coagulation (DIC) with elevated white blood cell count, elevated C-reactive protein (CRP), elevated D-dimer, low platelets and pyuria. The patient expired within one day. The authors attributed the acute coronary thrombosis in the nonculprit vessel to have been triggered by progression of the inflammatory process related to urosepsis complicated by DIC, increased CRP, and persistent shock [9].

Another similar case report published in 2015 by Wolny et al. $[10,11]$ who described a case of a 50 -year-old male, heavy smoker, known hypertensive and dyslipidemic, who presented with recurrent chest pain and ST segment elevation in II, III, and aVF. The patient received loading doses of dual antiplatelets and unfractionated heparin in the pre-hospital phase. Coronary angiogram showed distal occlusion of the RCA, TIMI 0 , and another significant long lesion in the proximal LAD with involvement of the first diagonal branch with TIMI 3 flow. Intravenous eptifibatide (bolus and continuous infusion) was started and the RCA was stented using two drug-eluting stents with good angiographic results and relief of symptoms. The lesion in the LAD was left untreated. After 2 hours, the patient had recurrence of chest pain and ECG showed new negative $T$ waves in the anterior leads. A second angiography showed acute occlusion of the $L A D$, distally to the significant lesion in the proximal segment. A drug-eluting stent was implanted in the proximal and medial segment of the LAD with good angiographic results. The patient was sent home within 6 days 10.

In our case, the symptom recurrence and second STEMI of the patient may have been a result of the hypotension due to LV dysfunction in the setting of ongoing myocardial inflammation peri-acute Ml. The exact mechanism of these events as well as the risk factors remain undetermined due to the few case reports and warrant further investigation.

\section{Conclusion}

Consecutive episodes of acute myocardial infarction in different coronary arteries occurring within hours to a few days after primary $\mathrm{PCl}$ for STEMI is uncommon and can be catastrophic if not detected early. The mechanism and predisposing factors to such event are still unclear. The optimum interventional treatment strategy regarding the immediate management of STEMI in patients with multi-vessel disease remains controversial.

\section{References}

1. O'Gara PT, Kushna FG, Ascheim DD, Casey DE Jr, Chung MK, et al. (2013) 2013 ACCF/AHA guideline for the management of STelevation myocardial infarction: Executive summary: A report of the American College of Cardiology Foundation/American Heart Association Task Force on Practice Guidelines. Circulation 127: 529-555.

2. Steg PG, James SK, Atar D, Badano LP, Blomstrom-Lundqvist C, et al. (2012) ESC guidelines for the management of acute myocardial infarction in patients presenting with ST-segment elevation. Eur Heart J 33: 2569-2619.

3. Wald DS, Morris JK, Wald NJ, Chase AJ, Edwards RJ, et al. (2013) Randomized trial of preventive angioplasty in myocardial infarction. N Engl J Med 369: 1115-1123.

4. Engstrøm T, Kelbæk $H$, Helqvist $S, H ø$ sten DE, Kløvgaard L, et al. (2015) Complete revascularisation versus treatment of the culprit lesion only in patients with ST-segment elevation myocardial infarction and multivessel disease (DANAMI-3PRIMULTI): An open-label, randomized controlled trial (DANAMI 3 PRIMulti Trial). The Lancet 386: 665-671.

5. Gershlick AH, Khan JN, Kelly DJ, Greenwood JP, Sasikaran T, et al. (2015) Randomized trial of complete versus lesion-only revascularization in patients undergoing primary percutaneous coronary intervention for STEMI and multivessel disease (The CvLPRIT Trial). J Am Coll Cardiol 65: 963-972.

6. Levine GN, Bates ER, Blankenship JC, Bailey SR, Bittl JA, et al. (2015) ACCF/AHA/SCAl focused update on primary percutaneous coronary intervention for patients with ST elevation myocardial infarction: An update of the 2011 ACCF/AHA/SCAI guideline for percutaneous coronary intervention and the 2013 ACCF/AHA guideline for the management of ST-elevation myocardial infarction. Circulation.

7. Kim MC, Jeong MH, Kim SH, Hong YJ, Kim JH, et al. (2014) Current status of coronary intervention in patients with STsegment elevation myocardial infarction and multivessel coronary artery disease. Korean Circ J 44930: 131-138.

8. Widimsky P, Holmes D (2011) How to treat patients with STelevation acute myocardial infarction and multi-vessel disease? Euro Heart J 32: 396-403.

9. Windecker S, Kolh P, Alfonso F, Collet JP, Cremer J, et al. (2014) 2014 ESC/EACTS Guidelines on myocardial revascularization. Eur Heart J.

10. Chang CT, Lu YH, Chu CY, Sheu SH, Hsu PC (2014) Two consecutive episodes of acute myocardial infarction occurring in different coronary arteries of a single patient with sepsis. Acta Cardiol Sin 30: 578-581.

11. Wolny R, Pregowski J, Bekta P, Chmielak Z, Witkowski A (2015) Early occlusion of the non-infarct related coronary artery following successful primary percutaneous coronary intervention in ST-elevation myocardial infarction. Postep Kardiol Inter 2: 136-140. 